\title{
Theoretical analysis and numerical verification of the consistency of viscous smoothed-particle-hydrodynamics formulations in simulating free-surface flows
}

\author{
Andrea Colagrossi, ${ }^{1, *}$ Matteo Antuono, ${ }^{2, \dagger}$ Antonio Souto-Iglesias, ${ }^{3, \dagger}$ and David Le Touzét, ${ }^{4,}$ \\ ${ }^{1}$ CNR-INSEAN, The Italian Ship Model Basin, Via di Vallerano 139, I-00128 ROMA, Italy and CESOS: Centre for Excellence for Ship and \\ Ocean Structures, NTNU, Trondheim, Norway \\ ${ }^{2}$ CNR-INSEAN, The Italian Ship Model Basin, Via di Vallerano 139, I-00128 ROMA, Italy \\ ${ }^{3}$ Naval Architecture Department (ETSIN), Technical University of Madrid (UPM), E-28040 Madrid, Spain \\ ${ }^{4}$ Fluid Mechanics Laboratory, Ecole Centrale de Nantes and CNRS, 1 rue de la Noë, Nantes, France
}

\begin{abstract}
The theoretical formulation of the smoothed particle hydrodynamics (SPH) method deserves great care because of some inconsistencies occurring when considering free-surface inviscid flows. Actually, in SPH formulations one usually assumes that (i) surface integral terms on the boundary of the interpolation kernel support are neglected, (ii) free-surface conditions are implicitly verified. These assumptions are studied in detail in the present work for free-surface Newtonian viscous flow. The consistency of classical viscous weakly compressible SPH formulations is investigated. In particular, the principle of virtual work is used to study the verification of the free-surface boundary conditions in a weak sense. The latter can be related to the global energy dissipation induced by the viscous term formulations and their consistency. Numerical verification of this theoretical analysis is provided on three free-surface test cases including a standing wave, with the three viscous term formulations investigated.
\end{abstract}

PACS number(s): 47.11.-j, 47.15.-x, 47.10.ad

\section{INTRODUCTION}

Free-surface flows are of interest in many engineering fields such as ocean and coastal engineering, hydraulics, oil and gas, process engineering, etc. In these flows the free-surface presence is a dominant part of the flow behavior. In particular, the nonlinear kinematic and dynamic conditions applying at this free boundary constitute a challenging part in the simulation of these flows. Simulation methods of free-surface flows are numerous and varied, depending mainly on the magnitude of the dynamics of the flow at hand. These methods range from, e.g., spectral methods in potential flow for slow-dynamics propagation of gravity waves, level-set or volume-of-fluid methods for fluid-body interactions, up to Lagrangian meshless methods for simulating violent flows implying large deformation of the free surface including fragmentations, reconnections, formation of jets and drops, etc., and intense impacts on partially immersed bodies.

The smoothed particle hydrodynamics (SPH) is a numerical method which has become widely applied to free-surface flows in recent years. Due its Lagrangian and meshless nature, its theoretical and numerical analysis is difficult and has not been addressed much in the literature. Among the few works available on this topic some deal with the general consistency and convergence of the method; see Mas-Gallic and Raviart [1], Di Lisio et al. [2,3], or Ben Moussa et al. [4]. Regarding more specifically the viscous free-surface flows at aim in the present work, a few papers address the consistency of SPH formulations of the viscous term; see, e.g., Español and Revenga [5] or Hu and Adams [6], but without the presence

\footnotetext{
*a.colagrossi@insean.it

${ }^{\dagger}$ m.antuono@insean.it

†̇antonio.souto@upm.es

¿david.letouze@ec-nantes.fr
}

of a free surface. Conversely, Colagrossi et al. [7] recently addressed the consistency of the SPH formulation in presence of a free surface, but for inviscid flow.

The objective of the present paper is thus to consider the consistency of the SPH formulations for a viscous flow in presence of a free surface. This consistency applies to two different aspects which are linked together: the implicit verification of the free-surface boundary conditions, especially the dynamic one, and the approximation of the viscous term of the Navier-Stokes equations, the consistency of the remaining terms having been studied already in [7] for an inviscid free-surface flow. These two aspects depend on the choice of the SPH formulation of the viscous stress tensor. In the present work the two most used SPH formulations are investigated, the one by Monaghan and Gingold [8], and the one by Morris et al. [9].

The methodology followed in the present investigation is first to analyze the theoretical consistency of the different viscous formulations once smoothing SPH operators are applied to the differential operators at the continuous level. In a second step it is checked on numerical test cases whether these theoretical findings hold after discretization. To perform the theoretical analysis we follow the same procedure as in [7], namely we study through the principle of virtual works the verification of the free-surface boundary conditions in a weak sense, and the consistency of the formulations. This consistency is analyzed both locally at the free surface and integrally over the domain in terms of viscous energy dissipation introduced.

The paper is divided as follows. In Secs. II and III the governing equations and related boundary conditions are introduced in the context of the SPH method. Then the approximation of the viscous stress tensor is studied in Sec. IV. We first introduce the principle of virtual works and the link is made between the smoothed viscous operator and the verification of the free-surface dynamic boundary condition. 
From this procedure we derive a new SPH formulation of the viscous term. Then the most classical SPH formulations of this term, respectively by Monaghan and Gingold [8] and by Morris et al. [9], are analyzed in detail, especially in terms of local convergence at the free surface. In Sec. V the global consistency of the different viscous formulations investigated is addressed in terms of correctness of the energy dissipated within the flow due to viscous stresses. Finally, in last Sec. VI the different theoretical findings of previous sections are checked after discretization on different numerical free-surface test cases including the widely studied standing wave problem.

\section{GOVERNING EQUATIONS}

\section{A. Field equations}

We place ourselves in the context of the so-called "weakly compressible" or "pseudocompressible" approach, which consists of modeling a compressible flow to simulate a problem in which the compressibility is negligible. In the context of free-surface flows this approach is classically adopted in SPH formulations. Actually, in truly incompressible formulations the pressure solution is obtained through an implicit method involving linear system solution, and requires one to impose the dynamic free-surface condition at the system boundary. In a meshless context the latter means to first detect the free surface, which is not an easy operation; see, e.g., [10]. Conversely, in weakly compressible Lagrangian formulations a fully explicit method is used and free-surface conditions are supposed to be implicitly verified. This assumption is strong and was studied in detail in [7] for inviscid flow.

Here we follow the methodology of [7] for viscous flow. Nonetheless, it must be emphasized that all the conclusions related to the viscous term itself in the present work do not depend on the weak-compressibility assumption and therefore stand for a truly-incompressible formulation as well.

The Navier-Stokes equations for a barotropic fluid in Lagrangian formalism read

$$
\left\{\begin{array}{l}
\frac{D \rho}{D t}+\rho \boldsymbol{\nabla} \cdot \boldsymbol{u}=0 \\
\frac{D u}{D t}=\boldsymbol{g}+\frac{\nabla \cdot \mathbb{T}}{\rho} \\
p=c_{0}^{2}\left(\rho-\rho_{0}\right),
\end{array}\right.
$$

where $\rho$ is the fluid density, $\rho_{0}$ is the reference density, $p$ is the pressure, $c_{0}$ is the reference sound velocity, and $g$ is external volume forces. The flow velocity $\boldsymbol{u}$ is defined as the material derivative of a fluid element position $\boldsymbol{r}$ :

$$
\frac{D r}{D t}=\boldsymbol{u} .
$$

$\mathbb{T}$ is the stress tensor of a Newtonian fluid:

$$
\mathbb{T}=(-p+\lambda \operatorname{tr} \mathbb{D}) \mathbb{1}+2 \mu \mathbb{D},
$$

with $\mathbb{D}$ being the rate of strain tensor, i.e., $\mathbb{D}=(\nabla \boldsymbol{u}+$ $\left.\nabla \boldsymbol{u}^{T}\right) / 2$. Finally, $\mu$ and $\lambda$ are the viscosity coefficients. For the analysis which follows, it is useful to consider the viscous part of the stress tensor:

$$
\mathbb{V}=\lambda \operatorname{tr} \mathbb{D} \mathbb{1}+2 \mu \mathbb{D} \text {. }
$$

The divergence of the stress tensor thus writes

$$
\begin{aligned}
\nabla \cdot \mathbb{T} & =-\nabla p+\nabla \cdot \mathbb{V} \\
& =-\nabla p+(\lambda+\mu) \nabla(\nabla \cdot \boldsymbol{u})+\mu \nabla^{2} \boldsymbol{u} .
\end{aligned}
$$

\section{Boundary conditions}

The previous field equations apply on a domain $\Omega$, which is bounded by $\partial \Omega$ composed of solid boundaries $\partial \Omega_{B}$ and free surfaces $\partial \Omega_{F}$. Two boundary conditions (BCs) apply on free surfaces of kinematic and dynamic nature. The kinematic freesurface BC is naturally verified in the Lagrangian formalism adopted. The dynamic free-surface BC (hereinafter DFSBC) expresses the continuity of stresses across the free surface. Assuming that surface tension is negligible, a "free" surface does not stand either normal stresses or tangential shear stresses. For a Newtonian fluid, by denoting such stress field as $\boldsymbol{t}$, the DFSBC reads

$$
\boldsymbol{t}=\mathbb{T} \cdot \boldsymbol{n}=(-p+\lambda \operatorname{tr} \mathbb{D}) \boldsymbol{n}+2 \mu \mathbb{D} \cdot \boldsymbol{n}=0,
$$

where $\boldsymbol{n}$ is the free-surface normal unit vector. After normal and tangential projections, considering that $\operatorname{tr} \mathbb{D}=\boldsymbol{\nabla} \cdot \boldsymbol{u}$ and $\boldsymbol{n} \cdot \mathbb{D} \cdot \boldsymbol{n}=\boldsymbol{n} \cdot \partial \boldsymbol{u} / \partial \boldsymbol{n}, \mathrm{Eq}$. (2.6) becomes

$$
\begin{gathered}
p=\lambda \nabla \cdot \boldsymbol{u}+2 \mu \boldsymbol{n} \cdot \partial \boldsymbol{u} / \partial \boldsymbol{n}, \\
\boldsymbol{\tau} \cdot \mathbb{D} \cdot \boldsymbol{n}=0,
\end{gathered}
$$

where $\tau$ is a unit vector of the free-surface tangent plane. As a consequence of Eq. (2.7) the pressure field is generally discontinuous across the free surface.

\section{CONTINUOUS SPH FORMULATION}

The SPH approximation of the field equations (2.1) is based on a smoothing based on a convolution integral over the fluid domain $\Omega$. A generic field $f$ is thus approximated by

$$
\langle f\rangle(\boldsymbol{r})=\int_{\Omega} f\left(\boldsymbol{r}^{\prime}\right) W\left(\boldsymbol{r}^{\prime}-\boldsymbol{r} ; h\right) d V^{\prime},
$$

where $W\left(\boldsymbol{r}^{\prime}-\boldsymbol{r} ; h\right)$ is a weight function which, in practical applications, has a compact support $\Omega(\boldsymbol{r})$ of characteristic length $h$ often referred to as the "smoothing length;" see Fig. 1.

1. A comprehensive review of the SPH method can be found in [11] for more detailed information.

The weight function $W\left(\boldsymbol{r}^{\prime}-\boldsymbol{r}, h\right)$, often referred to as "kernel," is positive, centered in $\boldsymbol{r}$, and monotonously decreases with the distance $s=\left|\boldsymbol{r}-\boldsymbol{r}^{\prime}\right|$. The kernel considered in this study is spherical, thus depending only on $s$. Hereinafter we adopt the notation $W\left(\boldsymbol{r}^{\prime}-\boldsymbol{r}\right)$, the dependence on $h$ being implicitly assumed. For Eq. (3.9) to be consistent (when $h \rightarrow 0$ ) the kernel $W$ must integrate to 1 ; see, e.g., [7]:

$$
\int_{\Omega} W\left(\boldsymbol{r}^{\prime}-\boldsymbol{r} ; h\right) d V^{\prime}=1 .
$$

Such a property is not satisfied when the kernel support is not fully included inside the fluid domain. This occurs for particles close to $\partial \Omega_{F}$; see Fig. 1. In that case approximation (3.9) is not consistent. 


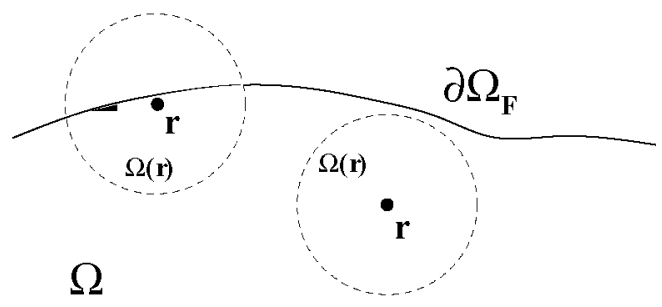

FIG. 1. (Color online) Configurations of the kernel support $\Omega(\boldsymbol{r})$ with respect to the fluid domain boundary.

This approximation for the gradient of a generic field $f$ reads

$$
\langle\nabla f\rangle(\boldsymbol{r})=\int_{\Omega} \nabla^{\prime} f\left(\boldsymbol{r}^{\prime}\right) W\left(\boldsymbol{r}^{\prime}-\boldsymbol{r}\right) d V^{\prime},
$$

with the prime in $\nabla^{\prime}$ denoting a derivative with respect to variable $\boldsymbol{r}^{\prime}$. Integrating by parts one gets

$$
\begin{aligned}
\langle\nabla f\rangle(\boldsymbol{r})= & \int_{\Omega} f\left(\boldsymbol{r}^{\prime}\right) \nabla W\left(\boldsymbol{r}^{\prime}-\boldsymbol{r}\right) d V^{\prime} \\
& +\int_{\partial \Omega} f\left(\boldsymbol{r}^{\prime}\right) W\left(\boldsymbol{r}^{\prime}-\boldsymbol{r}\right) \boldsymbol{n}^{\prime} d S^{\prime},
\end{aligned}
$$

with $\boldsymbol{n}^{\prime}$ a unit vector normal to $\partial \Omega$ pointing outwards $\Omega$, and where the sphericity of the kernel was used: $\nabla^{\prime} W\left(\boldsymbol{r}-\boldsymbol{r}^{\prime}\right)=-\nabla W\left(\boldsymbol{r}-\boldsymbol{r}^{\prime}\right)$. Note that the derivation now applies to the kernel function, which is known analytically, permitting us to access an approximation of $\nabla f$ from the knowledge of $f$. Further details can be found in [7] where an in-depth analysis of the smoothed differential operators and the surface integrals is provided.

When the smoothing procedure is applied to the differential operators of Eq. (2.1), the weakly compressible SPH continuous formulation of the Navier-Stokes equations is obtained:

$$
\left\{\begin{array}{l}
\frac{D \rho}{D t}+\rho\langle\nabla \cdot \boldsymbol{u}\rangle=0, \\
\frac{D u}{D t}=\boldsymbol{g}-\frac{\langle\nabla p\rangle}{\rho}+\frac{\langle\nabla \cdot \mathbb{V}\rangle}{\rho}, \\
p=c_{0}^{2}\left(\rho-\rho_{0}\right), \\
\frac{D r}{D t}=\boldsymbol{u},
\end{array}\right.
$$

where the notation $\langle f\rangle(\boldsymbol{r})$ has been shortened into $\langle f\rangle$. As shown in [7] for inviscid flow, the compatibility between the approximation formulas chosen in the momentum and mass conservation equations ensures global energy conservation of the system. This energy consistency can be investigated through the verification of the principle of virtual work (PVW). In the presence of a free surface, global energy conservation means that no energy gain or loss occurs through that "free" surface, which is coherent with it not being submitted to any stress. In that case the DFSBC is thus verified in a weak sense. In the present work we extend this analysis to viscous flow.

\section{SMOOTHED VISCOUS TERM}

In SPH-related literature the smoothed viscous term is modeled in many different ways. In the following paragraph, we first introduce the general structure of the viscous term in the SPH formalism through the PVW. Then, we focus on two of the most used formulations, namely (i) the Monaghan and Gingold [8] formulation, (ii) the Morris et al. formulation [9].

\section{A. Derivation of the smoothed viscous term through the principle of virtual work}

The principle of virtual works expresses the equality between the work of the internal forces and the one of the external forces due to the virtual displacement field $\delta \vec{w}$. In its general form it reads (see, e.g., $[12,13]$ )

$$
\begin{gathered}
\underbrace{\int_{\partial \Omega} \mathbb{T} \cdot n \cdot \delta \boldsymbol{w} d S}_{(1)}+\underbrace{\int_{\Omega} \rho\left(f-\frac{D u}{D t}\right) \cdot \delta \boldsymbol{w} d V}_{(2)} \\
=\underbrace{\int_{\Omega} \mathbb{T}: \mathbb{D}(\delta \boldsymbol{w}) d V}_{(3)} .
\end{gathered}
$$

The first two terms (1) and (2) represent the work of the stress tensor respectively on the fluid boundary and in the fluid domain. Their difference produces a variation of the internal energy (3) due to the virtual displacement field. The balance of the three integral terms guarantees the conservation of both the linear and angular momenta [14], and global energy conservation of the system. In the present analysis we are not interested in the work due to the presence of solid boundaries which is therefore considered equal to zero. By essence, on the free surface $\partial \Omega_{F} \mathbb{T} \mathbf{n}=0$, and $\rho(\mathbf{g}-D \mathbf{u} / D t)=-\nabla \cdot \mathbb{T}$. Thus expression (4.14) becomes

$$
-\underbrace{\int_{\Omega}(\boldsymbol{\nabla} \cdot \mathbb{T}) \cdot \delta \boldsymbol{w} d V}_{(2)}=\underbrace{\int_{\Omega} \mathbb{T}: \mathbb{D}(\delta \boldsymbol{w}) d V}_{(3)},
$$

which expresses the balance between the work of the stress tensor and the variation of the internal energy, both inside the domain. Thus to satisfy the DFSBC (2.6) in a weak sense, i.e., integrally, within the SPH scheme, it is sufficient to verify the equality (4.15) using the smoothed operators instead of the ordinary ones:

$$
-\int_{\Omega}\langle\nabla \cdot \mathbb{T}\rangle \cdot \delta \boldsymbol{w} d V=\int_{\Omega} \mathbb{T}:\langle\mathbb{D}(\delta \boldsymbol{w})\rangle d V
$$

In this case, no condition is explicitly enforced on that surface. After Eq. (2.3) is substituted into Eq. (4.15) the viscous and the pressure components can be treated separately. The latter ones were already discussed in [7], so only the viscous ones are considered in the following. Equation (4.15) now reads

$$
-\int_{\Omega}\langle\nabla \cdot \mathbb{V}\rangle \cdot \delta \boldsymbol{w} d V=\int_{\Omega} \mathbb{V}:\langle\mathbb{D}(\delta \boldsymbol{w})\rangle d V
$$


To ensure conservation of the angular momentum, the smoothed operator $\langle\mathbb{D}\rangle$ can be evaluated as (see [14])

$$
\begin{aligned}
\langle\mathbb{D}(\delta \boldsymbol{w})\rangle= & \frac{1}{2} \int_{\Omega}\left[\left(\delta \boldsymbol{w}^{\prime}-\delta \boldsymbol{w}\right) \otimes(\mathbb{L} \cdot \nabla W)\right. \\
& \left.+(\mathbb{L} \cdot \nabla W) \otimes\left(\delta \boldsymbol{w}^{\prime}-\delta \boldsymbol{w}\right)\right] d V^{\prime}, \\
\mathbb{L}= & {\left[\int_{\Omega}\left(r-r^{\prime}\right) \otimes \nabla W d V^{\prime}\right]^{-1}, }
\end{aligned}
$$

where $\mathbb{L}$ is a renormalization matrix which guarantees that $\langle\mathbb{D}(\delta \boldsymbol{w})\rangle$ is identically zero if $\delta \boldsymbol{w}$ is a pure rotation. Substituting Eq. (IV A) into Eq. (4.17) and following the procedure shown in [7], we get the smoothed operator:

$$
\langle\nabla \cdot \mathbb{V}\rangle^{\mathrm{PVW}}=\int_{\Omega}\left(\mathbb{L}^{\prime} \mathbb{V}^{\prime}+\mathbb{L} \mathbb{V}\right) \cdot \nabla W d V^{\prime}
$$

Since Eq. (4.19) has been derived from the PVW, it represents a natural way to approximate the viscous term of the governing equations (3.13), ensuring the conservation of the linear and angular momenta and the verification of the DFSBC in a weak sense. From a practical point of view, such a formulation is quite demanding in terms of CPU time since it requires a double integration and involves matrix operations. Nonetheless, as shown in the last section of the paper, the use of the formulation (4.19), hereinafter PVWF, permits us to get a higher accuracy with respect to the other viscous formulations.

\section{B. Monaghan and Gingold formulation}

Assuming that the viscosity coefficients are constant all over the fluid domain, the continuous Monaghan and Gingold formulation (hereinafter MGF) of the viscous term is

$$
\begin{aligned}
& \langle\nabla \cdot \mathbb{V}\rangle^{\mathrm{MG}}(\boldsymbol{r}) \\
& =\mu K \int_{\Omega} \frac{\left[\boldsymbol{u}\left(\boldsymbol{r}^{\prime}\right)-\boldsymbol{u}(\boldsymbol{r})\right] \cdot\left(\boldsymbol{r}^{\prime}-\boldsymbol{r}\right)}{\left|\boldsymbol{r}^{\prime}-\boldsymbol{r}\right|^{2}} \nabla W\left(\boldsymbol{r}^{\prime}-\boldsymbol{r}\right) d V^{\prime},
\end{aligned}
$$

where $K$ is a parameter depending on the spatial dimension ( $K=6,8,10$, respectively, in one, two, and three dimensions). Using the relations found by Español and Revenga [5] for the estimation of the second derivatives in SPH, inside the domain this formulation is consistent as

$$
\lim _{h \rightarrow 0}\langle\nabla \cdot \mathbb{V}\rangle^{\mathrm{MG}}=\mu \nabla^{2} \boldsymbol{u}+2 \mu \nabla(\nabla \cdot \boldsymbol{u}) .
$$

At the free surface, this is a priori not true anymore since the kernel support is incomplete, so that surface integrals do not vanish in the approximations of the differential operators such as Eq. (3.12). It is known that in such situations uncorrected SPH approximations of first derivatives, such as Eq. (3.12), are generally nonconsistent. Especially, it is proved in [7] that the pressure gradient formulation classically used in SPH solvers is neither convergent nor divergent at the free surface, whereas the velocity divergence is linearly convergent at the free surface for inviscid flow. Since the present work deals with viscous flows such an analysis must thus be performed for the second-order derivative of the velocity field. To study the consistency of the formulations of this viscous term at the free surface, we thus first follow the procedure proposed by [5] in taking into account the incompleteness of the kernel support and the presence of surface integrals. Then we analyze the consequences on the local and global consistency of the viscous SPH formulations studied.

\section{Taylor expansion of the Monaghan and Gingold formulation}

We follow here the procedure by Español and Revenga [5] applied to the MGF in introducing different tensors which will be useful for analysis of consistency at the free surface. Thanks to the kernel isotropy its gradient can be written in the compact form (4.22):

$$
\nabla W\left(\boldsymbol{r}^{\prime}-\boldsymbol{r}\right)=-\frac{\boldsymbol{r}^{\prime}-\boldsymbol{r}}{s} \frac{\partial W}{\partial s}, \quad s=\left|\boldsymbol{r}^{\prime}-\boldsymbol{r}\right| .
$$

If the origin of the frame of reference is set at $\boldsymbol{r}$, the integral (4.20) takes the following compact form:

$$
\langle\nabla \cdot \mathbb{V}\rangle^{\mathrm{MG}}=-\mu K \int_{\Omega} \frac{\left(\boldsymbol{u}^{\prime}-\boldsymbol{u}\right) \cdot \boldsymbol{r}^{\prime}}{\left|\boldsymbol{r}^{\prime}\right|^{3}} \boldsymbol{r}^{\prime} \frac{\partial W}{\partial s} d V^{\prime}
$$

where $\boldsymbol{u}(\boldsymbol{r})$ and $\boldsymbol{u}\left(\boldsymbol{r}^{\prime}\right)$ have been respectively shortened into $\boldsymbol{u}$ and $\boldsymbol{u}^{\prime}$. A Taylor expansion of the velocity field is performed:

$$
\boldsymbol{u}^{\prime}-\boldsymbol{u}=\left.\nabla \boldsymbol{u}\right|_{r} \cdot \boldsymbol{r}^{\prime}+\left.\frac{1}{2} \boldsymbol{r}^{\prime} \cdot \mathbb{H}\right|_{\boldsymbol{r}} \cdot \boldsymbol{r}^{\prime}+\mathcal{O}\left(\left|\boldsymbol{r}^{\prime}\right|^{3}\right)
$$

in which $\mathbb{H} \mid{ }_{r}$ denotes the Hessian tensor:

$$
\left[\left.\mathrm{HI}\right|_{r}\right]_{i j k}=\frac{\partial^{2} \boldsymbol{u}_{i}}{\partial \boldsymbol{r}_{j} \partial \boldsymbol{r}_{k}}
$$

Hereinafter $\left.\boldsymbol{\nabla} \boldsymbol{u}\right|_{r}$ is shortened into $\boldsymbol{\nabla} \boldsymbol{u}$ and $\left.\mathbb{H}\right|_{r}$ into $\mathbb{H}$. From Eqs. (4.23) and (4.24) comes

$$
\begin{aligned}
\frac{\left\langle\nabla \cdot \mathbb{V}^{\mathrm{MG}}\right\rangle}{\mu}= & -K \int_{\Omega} \nabla \boldsymbol{u}: \frac{\boldsymbol{r}^{\prime} \otimes \boldsymbol{r}^{\prime}}{\left|\boldsymbol{r}^{\prime}\right|^{3}} \boldsymbol{r}^{\prime} \frac{\partial W}{\partial s} d V^{\prime} \\
& -\frac{K}{2} \int_{\Omega} \mathbb{H}: \frac{\boldsymbol{r}^{\prime} \otimes \boldsymbol{r}^{\prime} \otimes \boldsymbol{r}^{\prime}}{\left|\boldsymbol{r}^{\prime}\right|^{3}} \cdot \boldsymbol{r}^{\prime} \frac{\partial W}{\partial s} d V^{\prime}+\mathcal{O}(h) .
\end{aligned}
$$

Since $r^{\prime} \otimes r^{\prime}$ is a symmetric tensor, the rate of deformation tensor $\mathbb{D}$ can substitute the gradient of the velocity inside the first integral of Eq. (4.26). Further, since $\mathbb{D}$ and $\mathbb{H}$ do not depend on $\boldsymbol{r}^{\prime}$, the relationship (4.26) can be written as

$$
\begin{aligned}
\frac{\langle\nabla \cdot \mathbb{V}\rangle^{M G}}{\mu}= & \mathbb{D}:\left[-K \int_{\Omega} \frac{\boldsymbol{r}^{\prime} \otimes \boldsymbol{r}^{\prime} \otimes \boldsymbol{r}^{\prime}}{\left|\boldsymbol{r}^{\prime}\right|^{3}} \frac{\partial W}{\partial s} d V^{\prime}\right] \\
& +\mathbb{H} \vdots\left[-\frac{K}{2} \int_{\Omega} \frac{\boldsymbol{r}^{\prime} \otimes \boldsymbol{r}^{\prime} \otimes \boldsymbol{r}^{\prime} \otimes \boldsymbol{r}^{\prime}}{\left|\boldsymbol{r}^{\prime}\right|^{3}} \frac{\partial W}{\partial s} d V^{\prime}\right] \\
& +\mathcal{O}(h) .
\end{aligned}
$$

The following two tensors can now be defined:

$$
\begin{gathered}
\mathbb{F}=-K \int_{\Omega} \frac{\boldsymbol{r}^{\prime} \otimes \boldsymbol{r}^{\prime} \otimes \boldsymbol{r}^{\prime}}{\left|\boldsymbol{r}^{\prime}\right|^{3}} \frac{\partial W}{\partial s} d V^{\prime} \\
\mathbb{G}=-\frac{K}{2} \int_{\Omega} \frac{\boldsymbol{r}^{\prime} \otimes \boldsymbol{r}^{\prime} \otimes \boldsymbol{r}^{\prime} \otimes \boldsymbol{r}^{\prime}}{\left|\boldsymbol{r}^{\prime}\right|^{3}} \frac{\partial W}{\partial s} d V^{\prime} .
\end{gathered}
$$

Consequently, the $i$ component of the smoothed viscous term (4.20) admits the following compact representation:

$$
\langle\nabla \cdot \mathbb{V}\rangle_{i}^{M G}=\mu\left[\mathbb{F}_{i j k} \mathbb{D}_{j k}+\mathbb{G}_{i j k l} \mathbb{H}_{j k l}\right]+\mathcal{O}(h) .
$$

With this form of the MGF its consistency can now be studied by using the properties of the tensors $\mathbb{F}$ and $\mathbb{G}$. 


\section{Local consistency of the Monaghan and Gingold formulation at the free surface}

As expected, if the considered material point is inside the domain, $\boldsymbol{r} \in \Omega$, from Eq. (4.29) one finds again the expression (4.21) found by Español and Revenga [5]; see Appendix A for details. Note that expression (4.21) is consistent with the continuous viscous stress definition (2.5) only if $\lambda=\mu$. This means that the MGF does not satisfy the Stokes hypothesis $(\lambda=-2 \mu / 3)$. The consequences of this fact have not received much attention in the literature and are left for future studies.

If the considered material point now belongs to the free surface, $\boldsymbol{r} \in \partial \Omega_{F}$, the value of the components of the tensor $\mathbb{G}$ are reduced due to the truncation of the kernel support; see Appendix A. Furthermore, the tensor $\mathbb{F}$ is not null anymore and becomes singular under certain circumstances, diverging like $h^{-1}$; see Appendix B. In this appendix it is also shown that even when the fluid is truly incompressible, such a singular behavior exists and it occurs when the normal component of the viscous stress vector is not identically zero, i.e., $\boldsymbol{n} \cdot \partial \boldsymbol{u} / \partial \boldsymbol{n} \not \equiv 0$.

\section{Morris et al. formulation}

The second classical expression of the viscous term considered in the present work is the Morris et al. [9] formulation (hereinafter MEAF). Its continuous expression reads

$$
\begin{aligned}
& \langle\nabla \cdot \mathbb{V}\rangle^{\mathrm{MEA}}(\boldsymbol{r}) \\
& \quad=2 \mu \int_{\Omega} \frac{\left(\boldsymbol{r}^{\prime}-\boldsymbol{r}\right) \cdot \nabla W\left(\boldsymbol{r}^{\prime}-\boldsymbol{r}\right)}{\left|\boldsymbol{r}^{\prime}-\boldsymbol{r}\right|^{2}}\left[\boldsymbol{u}\left(\boldsymbol{r}^{\prime}\right)-\boldsymbol{u}(\boldsymbol{r})\right] d V^{\prime} .
\end{aligned}
$$

Using the Taylor expansion (4.24) in the same way as for the MGF leads to

$$
\langle\nabla \cdot \mathbb{V}\rangle_{i}^{\mathrm{MEA}}=\mu\left[\nabla \boldsymbol{u}_{i j} \mathbb{N}_{j}+\mathbb{H}_{i j k} \mathbb{M}_{j k}\right]+\mathcal{O}(h),
$$

where

$$
\mathbb{N}_{j}=-2 \int_{\Omega} \frac{\boldsymbol{r}_{j}^{\prime}}{s} \frac{\partial W}{\partial s} d V^{\prime}, \quad \mathbb{M}_{j k}=-\int_{\Omega} \frac{\boldsymbol{r}_{j}^{\prime} \boldsymbol{r}_{k}^{\prime}}{s} \frac{\partial W}{\partial s} d V^{\prime}
$$

Similarly to the MGF, $\mathbb{N}$ is zero inside the fluid domain while it is equal to $-C / h \boldsymbol{n}$ at the free surface, where $C$ is a constant depending on the kernel function. The MEAF is thus also locally inconsistent at the free surface, diverging linearly.
For what concerns the second-order terms, $\mathbb{M}_{j k}=\delta_{j k}$ inside the fluid while it halves at the free surface, independently of the kernel shape. Then, inside the fluid domain the MEAF is consistent as

$$
\lim _{h \rightarrow 0}\langle\nabla \cdot \mathbb{V}\rangle^{\mathrm{MEA}}=\mu \nabla^{2} \boldsymbol{u}
$$

This means that, inside the domain, the MEAF approximates the exact viscous term for incompressible flows while the MGF also takes into account the weak-compressibility effects, but. not respecting the Stokes hypothesis (cf. Sec. B2).

At this stage we have established that both MGF and MEAF are consistent inside the domain. Conversely, due to the incompleteness of the kernel support, these formulations are, not surprisingly (cf. Sec. B), locally not consistent at the free surface, and both diverge linearly. However, this does not prejudge the global consistency of these formulations for free-surface flows, as will be shown in next sections.

\section{GLOBAL CONSISTENCY OF THE THREE VISCOUS FORMULATIONS AT HAND}

In the previous sections it has been shown that the different. $\mathrm{SPH}$ viscous term formulations are locally inconsistent at the free surface. In the present section we investigate their global consistency. This global consistency can be checked in different ways. A possibility is to monitor the convergence of the formulations toward reference solutions on viscous flow test cases for typical flow quantity evolutions. In that sense the consistency of SPH viscous formulations on typical internal flows such as Couette, Poiseuille, lid-driven cavity flows, etc., has been widely studied in the literature. The same will be performed in next section but for free-surface test cases.

Another possibility is to monitor the dissipation introduced by the smoothed viscous terms. This dissipation is quantified by the following integral:

$$
\int_{\Omega}\langle\nabla \cdot \mathbb{V}\rangle \cdot \boldsymbol{u} d V
$$

From Eq. (4.17) it follows that any formulation of the viscous term verifying the principle of virtual works, as the PVWF, will introduce the correct viscous dissipation during the flow evolution. From a theoretical point of view, the expression (5.34) can be integrated by parts to give a boundary term which is associated to the power of the surface forces and a bulk term
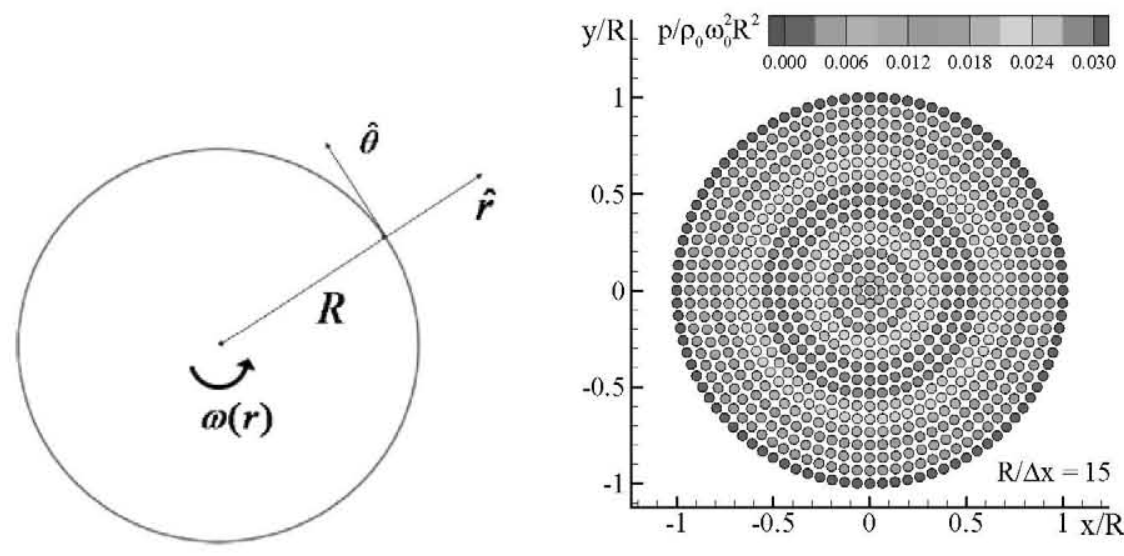

FIG. 2. (Color online) Configuration (left) and SPH setup and initial pressure for the test case in Sec. VI A. 

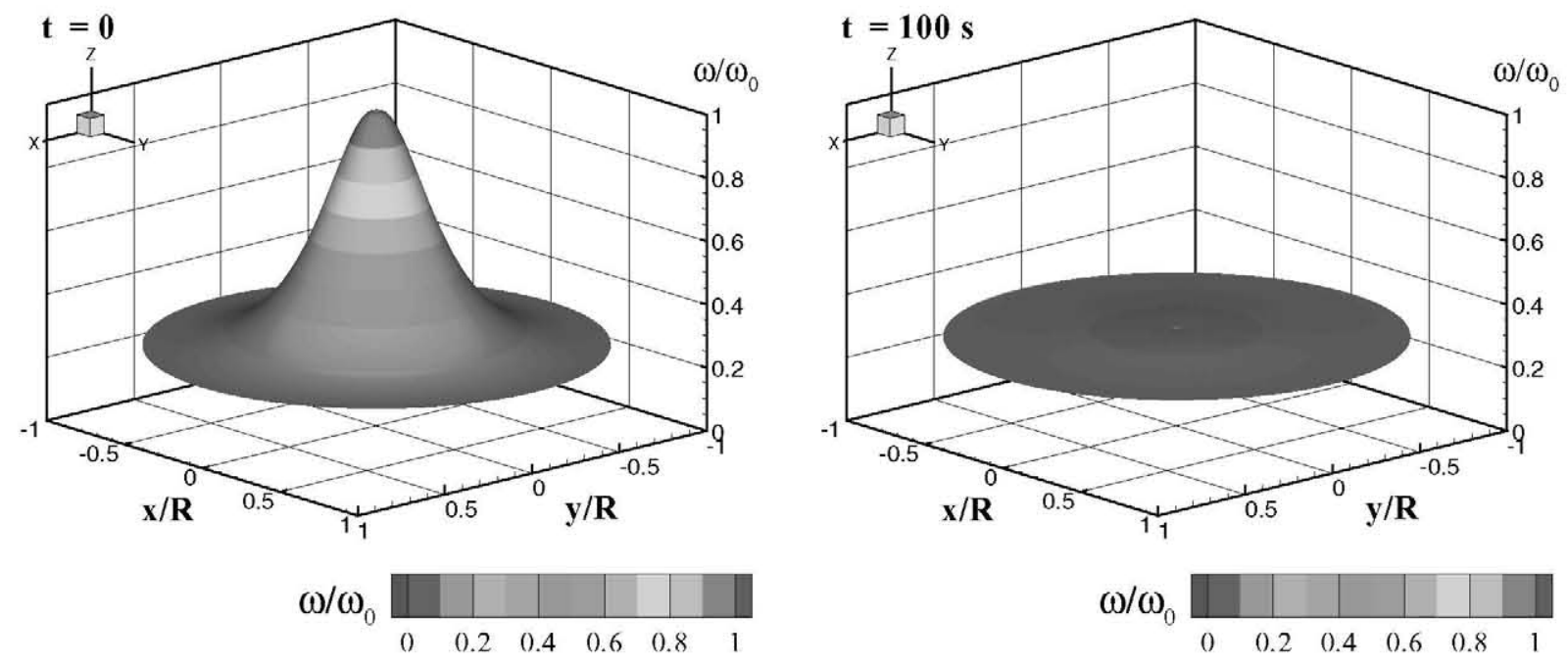

FIG. 3. (Color online) Evolution of a circular patch of fluid with a nonuniform initial vorticity distribution. Left: initial angular velocity $\omega$. Right: angular velocity $\omega$ at time $t=100 \mathrm{~s}$ predicted by the SPH simulation using the MGF. The contour levels are representative of the intensity of the vorticity field.

which, for the second principle of thermodynamics, is never negative and therefore causes the loss of kinetic energy of the fluid body.

If one considers a free-surface flow with no other boundary the following expressions can be derived (see Appendix C):

$$
\begin{aligned}
& \int_{\Omega}\langle\nabla \cdot \mathbb{V}\rangle^{\mathrm{MG}} \cdot \boldsymbol{u} d V=-\int_{\Omega} \mathbb{V}: \mathbb{D} d V+\mathcal{O}(h), \\
& \int_{\Omega}\langle\nabla \cdot \mathbb{V}\rangle^{\mathrm{MEA}} \cdot \boldsymbol{u} d V=-\mu \int_{\Omega}\|\nabla \boldsymbol{u}\|^{2} d V+\mathcal{O}(h) .
\end{aligned}
$$

From this result we can draw different interesting conclusions. First, both the integrals for the MGF and the MEAF are convergent, despite the local singularity of these formulations at the free surface. Second, as shown by Eq. (5.36), the dissipation associated to the MEAF is different from zero even in the case of a pure rigid rotation (that is, $\mathbb{D} \equiv 0$ ) where there should be no dissipation at all. Last, by choosing $\boldsymbol{u}$ as virtual displacement field into Eq. (5.35) it follows that the MGF satisfies the principle of virtual works (4.17) with an error of order $\mathcal{O}(h)$.

Summarizing, despite their local inconsistency at the free surface, PVWF and MGF are theoretically globally consistent for free-surface flow. Conversely, the MEAF is globally convergent but not to the proper viscous dissipation and is likely to be discarded for free-surface flow.

\section{TEST CASES}

In the present section we show to what extent the theoretical conclusions drawn in the previous sections with respect to the continuous smoothed viscous term remain valid after discretization. To this purpose three free-surface flow test cases were selected. The numerical parameters used in these simulations are (i) use of a renormalized Gaussian kernel, see, e.g., [15], with $3 h$ kernel support radius, (ii) $h / \Delta x=4 / 3$, with $\Delta x$ the mean particle interspace. In the simulations of this section the three formulations discussed in the paper are compared: (i) PVWF, Eq. (4.19), (ii) MGF, Eq. (4.20), and (iii) MEAF, Eq. (4.30). For each test case the results obtained through the different viscous models are compared with analytical or reference solutions.

\section{A. Evolution of a circular patch of fluid with a nonuniform initial vorticity distribution}

A fluid circular cylinder of radius $R=1$ subjected to a radial force field is considered (Fig. 2). The origin of the frame of reference is the cylinder center and the radial force is $g=-\beta^{2} r \hat{r}$ where $\beta$ is a constant parameter, $r$ is the radial coordinate, and $\hat{\boldsymbol{r}}$ is the radial unit vector. The angular coordinate is denoted by $\theta$ while $\hat{\boldsymbol{\theta}}$ indicates the tangential unit vector. The subscripts $r$ and $\theta$ are used to denote, respectively, the radial and angular components of the velocity field.

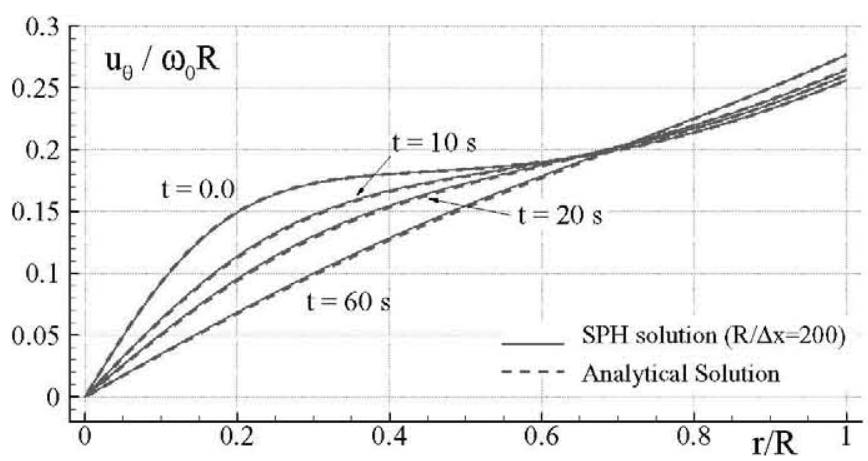

FIG. 4. (Color online) Evolution of a circular patch of fluid with a nonuniform initial vorticity distribution. Comparison between the velocity component $u_{\theta}$ predicted by the SPH simulation using the MGF (solid line), and the analytical solution (dashed line) at different times. 
An incompressible isotropic solution is sought. Under these hypotheses the Navier-Stokes equations reduce to

$$
\left\{\begin{array}{l}
-\frac{u_{\theta}^{2}}{r}=-\frac{1}{\rho_{0}} \frac{\partial p}{\partial r}-\beta^{2} r, \\
\frac{\partial u_{\theta}}{\partial t}=v\left\{\frac{1}{r} \frac{\partial}{\partial r}\left(r \frac{\partial u_{\theta}}{\partial r}\right)-\frac{u_{\theta}}{r^{2}}\right\} .
\end{array}\right.
$$

Since $\omega(r, t)=u_{\theta}(r, t) / r$, the second equation can be rearranged in the following format:

$$
\frac{\partial \omega}{\partial t}=\frac{v}{r}\left\{3 \frac{\partial \omega}{\partial r}+r \frac{\partial^{2} \omega}{\partial r^{2}}\right\} .
$$

The initial distribution for the angular velocity field is chosen as

$$
\omega(r, 0)=\omega_{0}\left\{\frac{l^{2}}{l^{2}+r^{2}}+2 \frac{l^{2} R}{\left(l^{2}+R^{2}\right)^{2}} r\right\},
$$

and does not depend on $\theta$ accordingly to the initial hypotheses. The constant parameters are $\ell^{2}=0.1 \mathrm{~m}^{2}, \omega_{0}=1 \mathrm{rad} / \mathrm{s}$, and $\beta=\pi / 8 \mathrm{~s}^{-1}$. The kinematic viscosity $v$ is equal to $10^{-3} \mathrm{~m}^{2} / \mathrm{s}$ and the Reynolds number is $\operatorname{Re}=R u_{\theta}(R, 0) / v=256$. With these values the velocity field actually remains independent of $\theta$ during the simulation.

In this configuration condition (2.8) is equivalent to $\partial \omega / \partial r=0$ at the boundary. Initial condition (6.39) actually satisfies this equality. Note that Eq. (6.38) is not coupled with the pressure equation and can therefore be solved numerically to get a reference solution for $\omega(r, t)$, hereinafter referred to as "analytical solution." Since there is no dependence on $\theta$, the symmetric part $\mathbb{D}$ and antisymmetric part $\mathbb{W}$ of the velocity gradient in polar coordinate are

$$
\begin{gathered}
\mathbb{D}=\frac{1}{2}\left(\begin{array}{cc}
0 & r \partial \omega / \partial r \\
r \partial \omega / \partial r & 0
\end{array}\right) \\
\mathbb{W}=\frac{1}{2}\left(\begin{array}{cc}
0 & -(2 \omega+r \partial \omega / \partial r) \\
2 \omega+r \partial \omega / \partial r & 0
\end{array}\right) .
\end{gathered}
$$

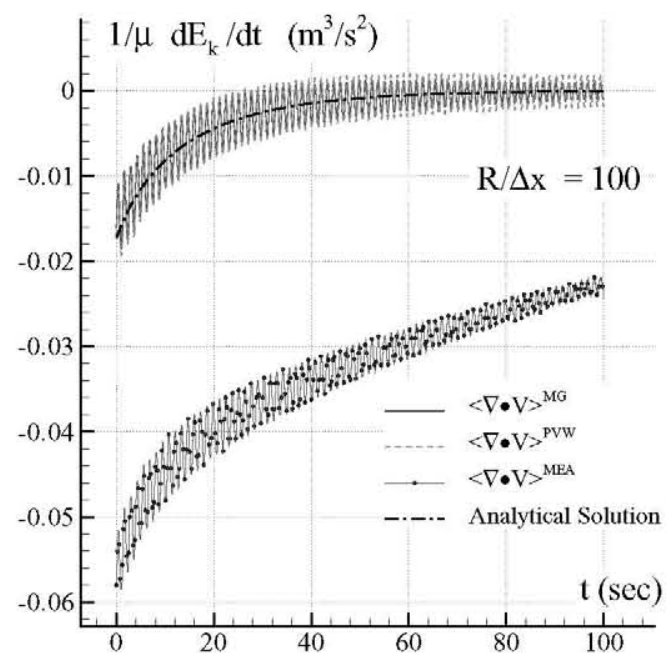

Since the domain always keeps circular, it is simple to prove that $\boldsymbol{n} \cdot \mathbb{D} \cdot \boldsymbol{n}=0$. This implies that the viscosity smooth operator $\langle\nabla \cdot \mathbb{V}\rangle^{\mathrm{MG}}$ is locally consistent also at the free surface in this situation, according to the results of Sec. IV B 2.

The initial setup of the SPH simulation is displayed in the right panel of Fig. 2. The left panel of Fig. 3 shows the initial angular velocity field and the related contour plot for the vorticity $\operatorname{curl}(u)=\omega+\frac{1}{2} r \partial \omega / \partial r$. Due to the viscous effects, for $t \rightarrow \infty$ the flow evolution theoretically converges to a rigid rotation with a constant angular velocity equal to $\omega \simeq 0.2848 \omega_{0}$. The right panel of Fig. 3 shows the angular velocity field predicted by the SPH simulation at time $t=100 \mathrm{~s}$ using the MGF. As can be seen in the right panel of Fig. 2, at the end of the simulation the initial nonuniform angular velocity field has been almost flattened by the viscosity action. The comparison between the analytical solution for $u_{\theta}$ (dashed lines) and the MGF SPH one (solid lines) at different times is presented in Fig. 4. A very close agreement is found and a linear profile is obtained for long times, which indicates that the motion has become a rigid rotation.

When using the PVWF the same results are found as when using the MGF. Conversely, the MEAF SPH simulation quickly diverges from the analytical solution.

Using Eq. (6.40) it is possible to evaluate the kinetic energy dissipation through

$$
\left.\frac{d E_{k}}{d t}=-2 \mu \int_{\Omega} \mathbb{D}: \mathbb{D}\right) d V=-\mu \int_{\Omega}(r \partial \omega / \partial r)^{2} d V .
$$

The left panel of Fig. 5 shows the evolution of $d E_{k} / d t$ evaluated with the analytical solution and through the SPH solver. In the latter case the three different formulations $\langle\nabla \cdot \mathbb{V}\rangle^{\mathrm{PVW}},\langle\nabla \cdot \mathbb{V}\rangle^{\mathrm{MG}},\langle\boldsymbol{\nabla} \cdot \mathbb{V}\rangle^{\mathrm{MEA}}$ were used. All the SPH simulations were performed with the same spatial resolution,

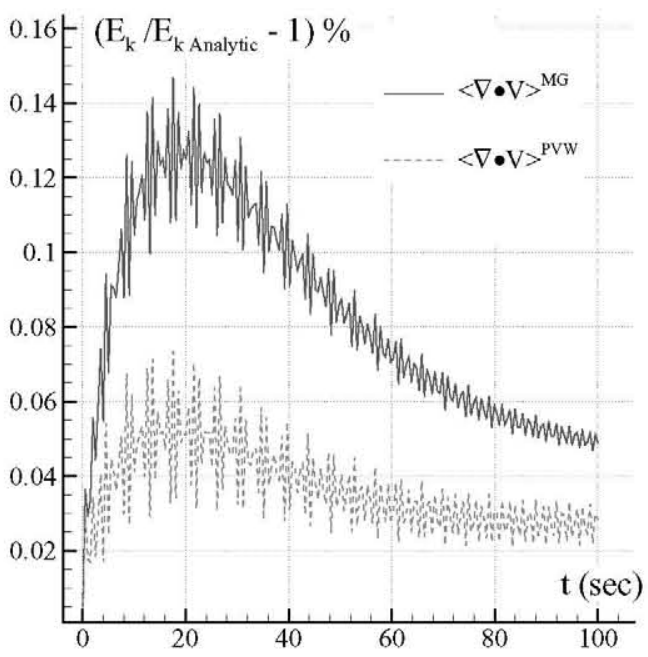

FIG. 5. (Color online) Evolution of a circular patch of fluid with a nonuniform initial vorticity distribution. Left: time histories of the kinetic energy dissipation $d E_{k} / d t$ evaluated using $\langle\nabla \cdot \mathbb{V}\rangle^{P V W},\langle\nabla \cdot \mathbb{V}\rangle^{\mathrm{MG}},\langle\nabla \cdot \mathbb{V}\rangle^{\mathrm{MEA}}$. The dashed-dotted line represents the analytical solution. Right: relative error on the kinetic energy evolution between the analytical solution and $\mathrm{SPH}$ solutions for $\langle\nabla \cdot \mathbb{V}\rangle^{\mathrm{PVW}}$ and $\langle\nabla \cdot \mathbb{V}\rangle^{\mathrm{MG}}$. 

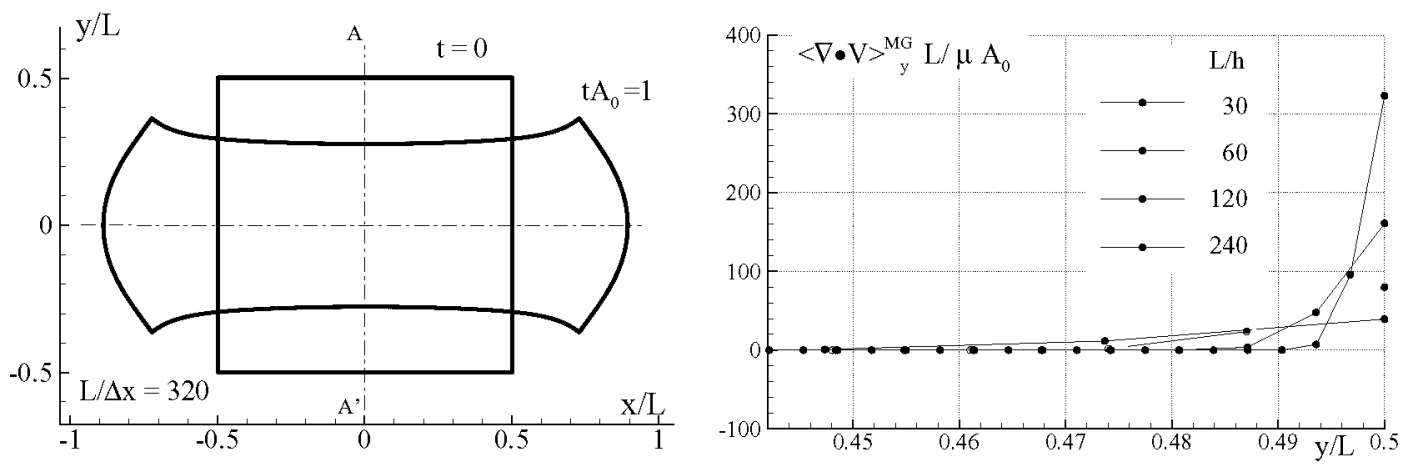

FIG. 6. (Color online) Stretching of a square patch of viscous fluid. Left: free surface configuration at time $t=0$ and $t A_{0}=1$. Right: enlarged view of the $y$-component of the vector $\langle\nabla \cdot \mathbb{V}\rangle^{M G}$ evaluated along the symmetry axis AA' at initial time $t=0$ for decreasing smoothing lengths $h$.

that is $R / \Delta x=100$. The first two formulations are in very good agreement with the analytical solution, but for high frequency oscillations related to the weak-compressibility assumption. Conversely, when the MEAF is used the kinetic energy dissipation is much higher. This result confirms the validity of the theoretical analysis made in the previous sections, also at the discrete level. In particular, the contribution of $\mathbb{W}: \mathbb{W}$ in Eq. (5.36) leads to an unphysical increase of the viscous dissipation for this MEA formulation. The right panel of Fig. 5 shows the time history of the relative error on the kinetic energy $E_{k}$ for the PVWF and MGF simulations. It confirms again that the preceding theoretical findings hold at the discrete level: the PVWF is actually more accurate than the MGF.

In the next subsection we show that even for a problem where the rate strain tensor $\mathbb{D}$ is predominant with respect to the tensor $\mathbb{W}$, i.e., the vorticity field is weak, the MEAF still provides unphysical values of the viscous dissipation.

\section{B. Stretching of a square patch of viscous fluid}

As a second test case we consider a square fluid domain $\Omega$ with side length $L$, subjected at $t=0$ to the velocity field:

$\left\{\begin{array}{l}u_{0}(x, y)=A_{0} x \\ v_{0}(x, y)=-A_{0} y\end{array} \quad \Rightarrow \mathbb{D}_{0}=\left(\begin{array}{cc}A_{0} & 0 \\ 0 & -A_{0}\end{array}\right), \quad \mathbb{W}_{0}=0\right.$

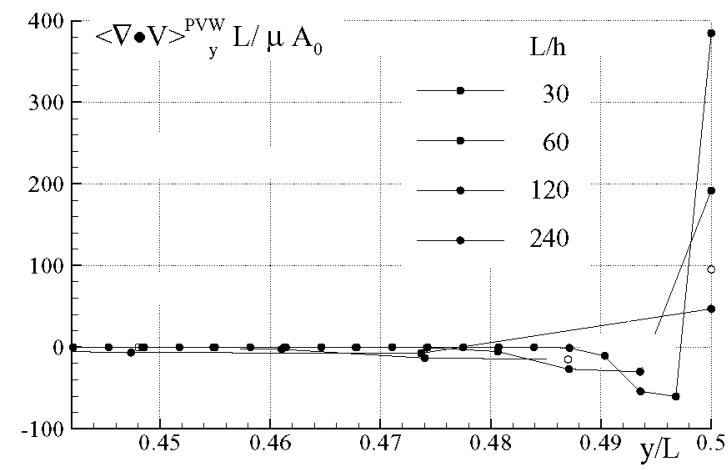

The square center is considered the origin of the frame of reference. Since no external force is considered, the velocity field $u_{0}$ tends to stretch the domain $\Omega$ along the $x$ axis and to contract it in the $y$ direction; see the left plot of Fig. 6. The initial velocity field satisfies the boundary condition $\tau \cdot \mathbb{D}_{0}$. $n=0$. The initial pressure field is evaluated by solving the Poisson equation for incompressible flow $\nabla^{2} p=-2 \rho A_{0}^{2}$; see, e.g., [16]. The pressure boundary condition along the free surface is $p=-2 \mu\left(n \cdot \mathbb{D}_{0} \cdot n\right)$ and the Reynolds number is $\operatorname{Re}=L^{2} A_{0} / v=20$. Due to this rather strong viscosity about $80 \%$ of the initial kinetic energy is dissipated in the interval $t A_{0}=[0,1]$. Since $n \cdot \mathbb{D} \cdot n$ is not null we expect the viscous smoothing operators $\langle\nabla, \mathbb{V}\rangle$ to be singular at the free surface. This is shown at initial time in the right panel of Fig. 6 and in Fig. 7, showing a linear divergence for all the three formulations. One can note that, conversely to the MGF and MEAF, the PVWF solutions present both negative and positive values near the free surface. This is likely due to the fact that this operator satisfies the integral equation (4.17). Unfortunately, this behavior induces large numerical instabilities in time, which leads to unphysical clumping of the particles close to the free surface. Due to these instabilities the operator $\langle\nabla \cdot V\rangle^{\text {PVW }}$ can be only used for limited time intervals.

For this test case no analytic solution can be derived and the SPH solutions are compared to a standard commercial volume of Fluid Finite volume solver (STAR-CCM+ here). In this reference solution the space resolution considered is

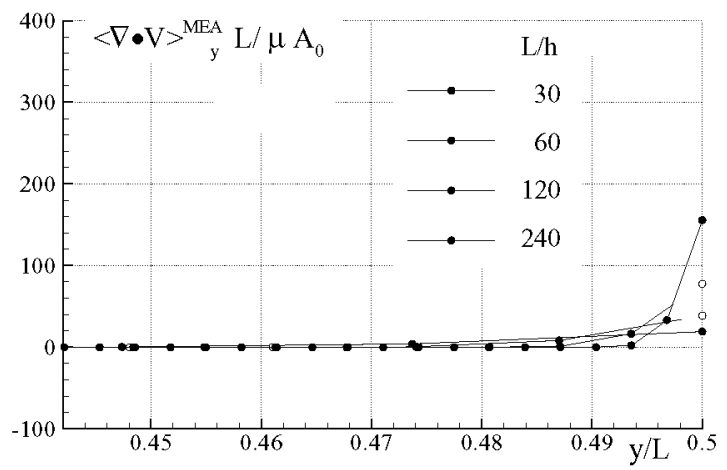

FIG. 7. (Color online) Stretching of a square patch of viscous fluid. Enlarged views of the $y$ component of the vectors $\langle\nabla \cdot \mathbb{V}\rangle^{\text {PVW }}($ left) and $\langle\nabla \cdot \mathbb{V}\rangle^{\mathrm{MEA}}$ (right) evaluated along the symmetry axis $A A^{\prime}$ at initial time $t=0$ for decreasing smoothing lengths $h$. 

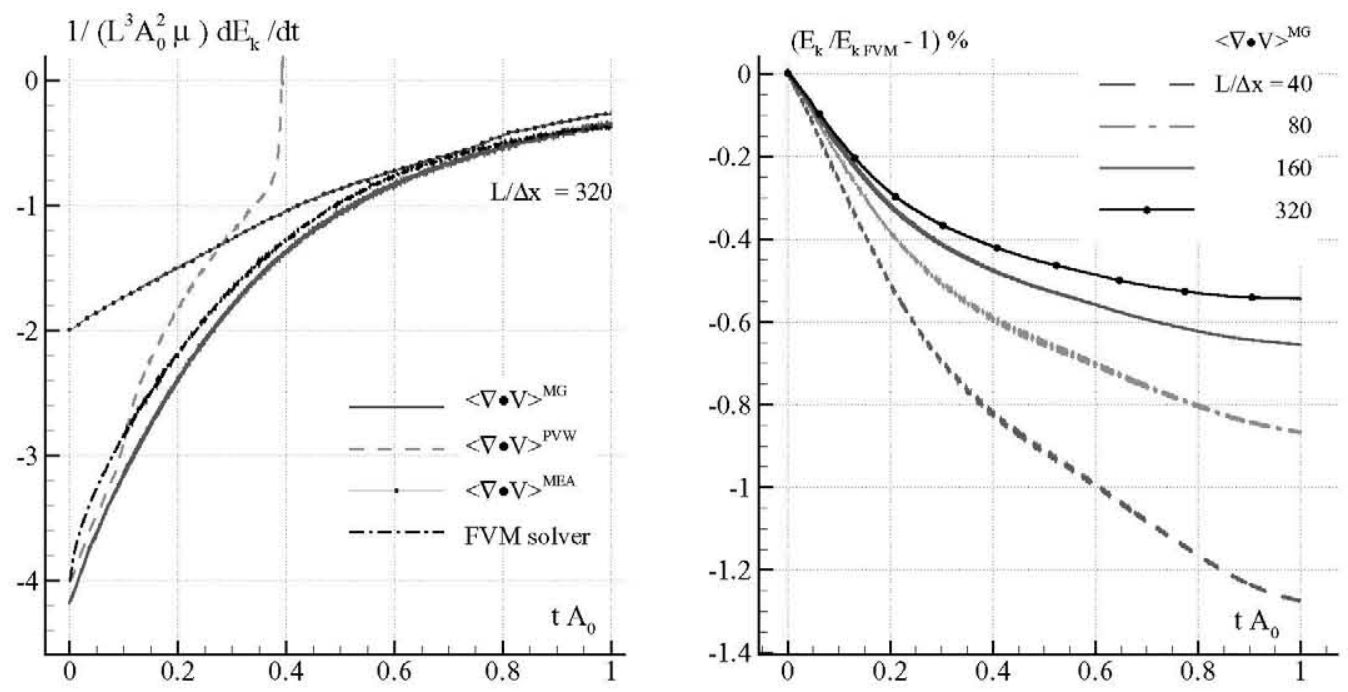

FIG. 8. (Color online) Stretching of a square patch of viscous fluid. Left: time histories of the kinetic energy dissipation $d E_{k} / d t$ for the three formulations. The dashed-dotted line represents the solution obtained through a finite-difference scheme solver. Right: relative error between the kinetic energy evaluated with the SPH MGF formulation and the one obtained by the FDM solver.

$L / \Delta x=320$. The left panel of Fig. 8 shows the time history of the kinetic energy dissipation. The kinetic energy dissipation at initial time $t=0$ is equal to $d E_{k} / d t=-4 \mu A_{0}^{2} L^{3}$. This value is very accurately obtained when $\langle\nabla \cdot \mathbb{V}\rangle^{P V W}$ is used, with a relative error of $0.04 \%$, against about $5 \%$ when the MGF is adopted. When using the MEA operator this initial value of $d E_{k} / d t$ is halved, as theoretically predicted by Eq. (5.36). Further during the evolution the MGF remains in good agreement with the FDM reference, whereas the PVWF performs well only up to $t=0.1 A_{0}$ before numerical instabilities develop up to stopping the simulation. In the right panel of the same figure is plotted the relative error between the MGF solution at different space resolutions and the FDM reference using the finest resolution $(L / \Delta x=320)$. A linear convergence is observed for the MGF SPH simulation and the relative error with the FDM for $L / \Delta x=320$ is about $0.55 \%$.

\section{Standing Wave}

As a last test case we investigate the widely studied standing wave problem. In this problem a periodic standing wave of wavelength $\lambda$ and wave amplitude $A$ is considered. The numerical domain chosen to study this problem is a domain of width $L=\lambda / 2$ with symmetry conditions applied on its vertical boundaries. The considered water filling height is $H=L$. The wave number is $k=2 \pi / \lambda$ and the wave steepness is $\epsilon=2 A / \lambda$.

For small-amplitude waves, i.e., $\epsilon<0.01$, in an inviscid context the potential theory predicts the following approximate solution:

$$
\begin{gathered}
\varphi(x, y, t)=\varphi_{0}(x, y) \cos (\omega t), \\
\varphi_{0}(x, y)=-\frac{A g}{\omega} \frac{\cosh [k(y+H)]}{\cosh (k H)} \cos (k x) .
\end{gathered}
$$

The angular frequency $\omega$ is given by the dispersion relation for gravity waves $\omega^{2}=g k \tanh (k H)$, with $g$ the gravity acceleration. A sketch of the problem is displayed in top left panel of Fig. 9. At time $t=0$ the free surface is horizontal and the time derivative of the velocity potential is zero in the whole fluid domain. As a consequence the pressure field at this time can be simply assumed to be hydrostatic with an error of $\mathcal{O}\left(\epsilon^{2}\right)$ while the initial fluid velocity is given by $\nabla \varphi_{0}$.

The potential theory predicts that the total energy of the standing wave is conserved in time. If the fluid is viscous, as it is considered here, it is still possible to obtain an approximate analytical solution, see Lighthill [17], which gives as decay of the kinetic energy:

$$
E_{\mathrm{kin}}(t)=\frac{\lambda A^{2} g}{8} e^{-4 v k^{2} t}[1+\cos (2 \omega t)] .
$$

In Fig. 9 the decay of the kinetic energy in time is plotted for the different SPH formulations of the viscous term. The Reynolds number is $\operatorname{Re}=\lambda U_{\max } / v=140$ where $U_{\max }$ is the maximum fluid velocity obtained from Eq. (6.43). All the SPH viscous formulations exhibit a linear convergence, and a converged solution is obtained for the finest resolution $H / \Delta x=240$. The PVWF gives the best agreement with the analytical solution (6.44) while the MGF presents a small (and almost negligible) underprediction of the damping rate. On the contrary, as for the other test cases the MEAF fails in predicting the proper kinetic energy dissipation of this free-surface problem. These conclusions further confirm that the theoretical findings of the present work hold after discretization.

\section{CONCLUSIONS}

In the present paper we followed the analysis made in [7] for inviscid free-surface flow but for a viscous flow here. Since at continuous level the lack of full support of the SPH kernel raises a consistency issue at the free surface, first studied was the local consistency of the viscous second-order differential operator at the free surface. This local consistency depends on the quantity to which the operator applies, with a link 

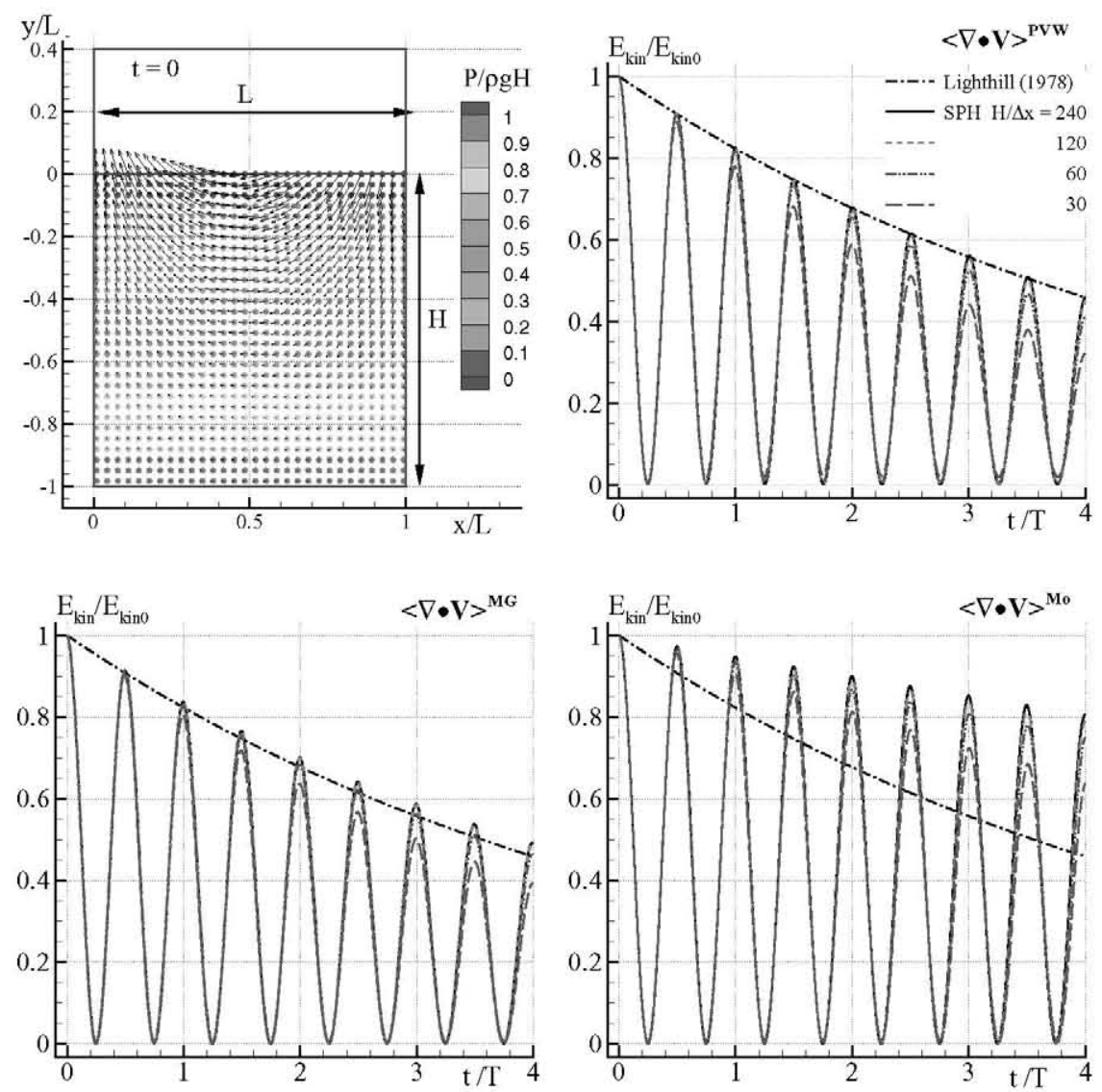

FIG. 9. (Color online) Standing wave: sketch of the problem (top left) and the decay of the kinetic energy as predicted by using the PVWF (top right), the MGF (bottom left), and the MEAF (bottom right). to the boundary condition considered. The conclusions of this analysis, for instance for the Monaghan and Gingold formulation [8], are that this second-order operator is not divergent at the free surface in its tangential component, and divergent of order 1 in its normal component.

It was then shown that this local inconsistency does not prejudge the global consistency since the latter is also linked to the compatibility between the different equations of the system (global energy conservation), which implies the verification of the boundary conditions in an integral sense. In practice, one is more interested in this global consistency than the local one. The conclusions drawn from this analysis at continuous level in the present paper are that the classical Monaghan and Gingold formulation of the viscous term can be used in the context of viscous free-surface flow, whereas it is not the case for the other classical formulation by Morris et al. [9].

When proceeding to the analysis of the compatibility of the system of equations, here achieved thanks to the principle of virtual works, it is also possible to derive new formulations which intrinsically verify the compatibility. This led us to propose a new formulation of the viscous term, referred to as PVW formulation. Both the Monaghan and Gingold and the PVW formulations permit us to verify implicitly the dynamic free-surface boundary condition in a weak sense.

Finally, the main question raised by such an analysis at continuous level for SPH practitioners is the applicability of its conclusions to numerical simulations of such viscous free-surface flows, i.e., after discretization. This question is even more critical if one recalls that discrete SPH first-order differential operators are divergent even inside the domain for an irregular distribution of the particles (see, e.g., [15]). To the purpose of answering this practical question different. numerical test cases were performed in the last part of the paper, including the classical standing-wave case. These test cases permit us to show that all the theoretical findings derived at a continuous level hold after discretization.

\section{ACKNOWLEDGMENTS}

The research leading to these results has received funding from the European Community's Seventh Framework Programme (FP7/2007-2013) under Grant Agreement No. 225967 "NextMuSE." This work was also partially supported by the Centre for Ships and Ocean Structures (CeSOS), NTNU, Trondheim, within the "Violent Water-Vessel Interactions and Related Structural Load" project, and by the Spanish Ministry for Science and Innovation under Grant No. TRA201016988, "Caracterización Numérica y Experimental de las Cargas Fluido-Dinámicas en el transporte de Gas Licuado." Finally, we give our sincere gratitude to Louis Delorme from EUROCOPTER-EADS for collaboration in the early part of this work.

\section{APPENDIX A: EVALUATION OF $\mathbb{G}$ IN THREE DIMENSIONS}

Let us consider a material point which is in the interior of the fluid domain $\Omega$. Using a spherical coordinate system 
centered at the particle position $r$ we get

$$
\int_{\Omega} W\left(\boldsymbol{r}^{\prime}-\boldsymbol{r}\right) d V=\int_{0}^{2 \pi} d \theta \int_{0}^{\pi} d \phi \int_{0}^{\infty} W(s) s^{2} \sin (\phi) d s=1
$$

since the kernel integral is equal to 1 by definition. It follows that

$$
\int_{0}^{\infty} s^{2} W(s) d s=\frac{1}{4 \pi}
$$

Taking into account relation (4.22) and integrating by parts Eq. (A2) comes

$$
\int_{0}^{\infty} s^{3} \frac{\partial W}{\partial s} d s=-\frac{3}{4 \pi}
$$

Using equalities (A2) and (A3), the tensor $\mathbb{G}$ defined in Sec. IV becomes

$$
\mathbb{G}=-\frac{K}{2} \int_{0}^{\pi} d \phi \int_{0}^{2 \pi} d \theta \int_{0}^{\infty}\left(\hat{\boldsymbol{r}^{\prime}} \otimes \hat{\boldsymbol{r}}^{\prime} \otimes \hat{\boldsymbol{r}}^{\prime} \otimes \hat{\boldsymbol{r}}^{\prime}\right) s^{3} \frac{\partial W}{\partial s} \sin (\phi) d s,
$$

where $s$ is the distance $\left|\boldsymbol{r}^{\prime}\right|$ and $\hat{\boldsymbol{r}}^{\prime}$ is the unit vector $[\cos \theta \sin \phi, \sin \theta \sin \phi, \cos \phi]$. Using relation (A3), expression (A4) can be rewritten as

$$
\mathbb{G}=K \frac{3}{8 \pi} \int_{0}^{\pi} d \phi \int_{0}^{2 \pi} d \theta\left(\hat{\boldsymbol{r}^{\prime}} \otimes \hat{\boldsymbol{r}}^{\prime} \otimes \hat{\boldsymbol{r}}^{\prime} \otimes \hat{\boldsymbol{r}}^{\prime}\right) \sin (\phi) .
$$

This result shows that the tensor $\mathbb{G}$ does not depend on the kernel function. Further, this tensor is symmetric and only 21 components are different from zero $(K=10$ in three dimensions):

$$
\begin{aligned}
\mathbb{G}[1,1,1,1] & =\mathbb{G}[2,2,2,2]=\mathbb{G}[3,3,3,3]=3, \\
\mathbb{G}[1,1,2,2]=\mathbb{G}[1,1,3,3]=\mathbb{G}[2,2,1,1]=\mathbb{G}[2,2,3,3]=\mathbb{G}[3,3,1,1] & =\mathbb{G}[3,3,2,2]=1, \\
\mathbb{G}[1,2,1,2]=\mathbb{G}[1,2,2,1]=\mathbb{G}[1,3,1,3]=\mathbb{G}[1,3,3,1]=\mathbb{G}[2,3,2,3] & =\mathbb{G}[2,3,3,2]=1, \\
\mathbb{G}[2,1,1,2]=\mathbb{G}[2,1,2,1]=\mathbb{G}[3,1,1,3]=\mathbb{G}[3,1,3,1]=\mathbb{G}[3,2,2,3]=\mathbb{G}[3,2,3,2] & =1 .
\end{aligned}
$$

Using the above results and some algebra $\mathbb{G}$ can be rewritten as $\mathbb{J}+\mathbb{A}$ where (see, e.g., [18])

$$
\mathbb{J}_{i j k l}=\delta_{i j} \delta_{k l} \quad \text { and } \quad \mathbb{A}_{i j k l}=\frac{\partial^{2}\left(\boldsymbol{r}_{i} \boldsymbol{r}_{j}\right)}{\partial \boldsymbol{r}_{k} \partial \boldsymbol{r}_{l}} .
$$

Consequently, when the fourth-order tensor $\mathbb{G}$ is applied to the third-order Hessian tensor $\mathbb{H}$ defined in Sec. IV, one obtains

$$
\mathbb{G}_{i j k l} \mathbb{H}_{j k l}=\left[\mathbb{J}_{i j k l} \mathbb{H}_{j k l}+\frac{\partial^{2}\left(\boldsymbol{r}_{i} \boldsymbol{r}_{j}\right)}{\partial \boldsymbol{r}_{k} \partial \boldsymbol{r}_{l}} \mathbb{H}_{j k l}\right] .
$$

For a point which is still in the interior of the fluid domain IF is equal to zero, as demonstrated in Appendix B. Consequently the smoothed viscous term (4.29) becomes

$$
\begin{aligned}
\langle\nabla \cdot \mathbb{V}\rangle_{i}^{M G} & =\mu \mathbb{G}_{i j k l} \mathbb{H}_{j k l}+\mathcal{O}(h) \\
& =\mu\left[\mathbb{J}_{i j k l} \mathbb{H}_{j k l}+\frac{\partial^{2}\left(\boldsymbol{r}_{i} \boldsymbol{r}_{j}\right)}{\partial \boldsymbol{r}_{k} \partial \boldsymbol{r}_{l}} \mathbb{H}_{j k l}\right]+\mathcal{O}(h),
\end{aligned}
$$

where

$$
\left[\left.\nabla^{2} \boldsymbol{u}\right|_{r}\right]_{i}=\mathbb{J}_{i j k l} \mathbb{H}_{j k l} \quad \text { and } \quad\left[\left.\nabla(\nabla \cdot \boldsymbol{u})\right|_{r}\right]_{i}=\frac{1}{2} \frac{\partial^{2}\left(\boldsymbol{r}_{i} \boldsymbol{r}_{j}\right)}{\partial \boldsymbol{r}_{k} \partial \boldsymbol{r}_{l}} \mathbb{H}_{j k l}
$$

It follows that

$$
\lim _{h \rightarrow 0}\langle\nabla \cdot \mathbb{V}\rangle^{\mathrm{MG}}=\mu \nabla^{2} \boldsymbol{u}(\boldsymbol{r})+2 \mu \nabla(\boldsymbol{\nabla} \cdot \boldsymbol{u})(\boldsymbol{r}) .
$$

Finally, due to the symmetry properties of the tensor $\mathbb{G}$, the smoothed viscous tensor can be written as

$$
\langle\mathbb{V}\rangle_{i j}^{\mathrm{MG}}=\mu \mathbb{G}_{i j k l} \mathbb{D}_{k l}+\mathcal{O}(h),
$$

and $\mu \mathbb{G}$ can be seen as the elasticity tensor used in the linear elasticity theory (e.g., see [19]).
If the material point is now on the free surface, that is, $r \in$ $\partial \Omega_{F}$, and if this surface is regular, $\partial \Omega_{F}$ can be approximated by its tangent plane in $\boldsymbol{r}$. As a consequence, integral (A5) has to be evaluated on the half space corresponding to the fluid region and the range of the zenith angle $\phi$ changes into $[\pi / 2, \pi]$. Then, we get

$$
\mathbb{G}=K \frac{3}{8 \pi} \int_{\pi / 2}^{\pi} d \phi \int_{0}^{2 \pi} d \theta\left(\hat{\boldsymbol{r}}^{\prime} \otimes \hat{\boldsymbol{r}}^{\prime} \otimes \hat{\boldsymbol{r}}^{\prime} \otimes \hat{\boldsymbol{r}}^{\prime}\right) \sin (\phi),
$$

and all the components of the tensor $\mathbb{G}$ are halved.

\section{APPENDIX B: SMOOTHED VISCOUS TERM SINGULARITY}

If one approximates a general velocity field to its linear component, expression (4.29) of the smoothed viscous term (4.20) reduces to

$$
\langle\nabla \cdot \mathbb{V}\rangle_{i}=\mu \mathrm{F}_{i j k} \mathbb{D}_{j k}
$$

Let us now assume as in Appendix A that $\boldsymbol{r}$ is a point of the free surface $\left(\boldsymbol{r} \in \partial \Omega_{F}\right)$ and that this surface is regular. Again, the free surface can be approximated by its tangent plane in $r$ in that case of normal unit vector $\boldsymbol{n}$ pointing outside the fluid and of tangential unit vector $\boldsymbol{\tau}$. The tensor $\mathbb{F}$ can then be evaluated by assuming a local frame of reference such that $\boldsymbol{n}=\boldsymbol{e}_{3}$ and by using a spherical coordinate system. Under these assumptions, integral (4.28) takes the following form:

$$
\begin{aligned}
& \mathbb{F}=-K\left[\int_{\pi / 2}^{\pi} d \phi \int_{0}^{2 \pi} d \theta\left[\hat{\boldsymbol{r}}^{\prime} \otimes \hat{\boldsymbol{r}}^{\prime} \otimes \hat{\boldsymbol{r}}^{\prime}\right] \sin (\phi)\right] \\
& \times\left[\int_{0}^{\infty} s^{2} \frac{\partial W}{\partial s} d s\right]
\end{aligned}
$$


in which $s$ is the distance $\left|\boldsymbol{r}^{\prime}\right|$, and $\hat{\boldsymbol{r}}^{\prime}$ the unit vector $[\cos \theta \sin \phi, \sin \theta \sin \phi, \cos \phi]$. Since the spherical support of the kernel is halved by the free-surface plane, the tensor $\mathbb{F}$ is not identically null. Actually, its components read

$$
\left\{\begin{array}{l}
\mathbb{F} \cdot \boldsymbol{e}_{1}=\frac{C}{h}\left(\boldsymbol{e}_{1} \otimes \boldsymbol{e}_{3}+\boldsymbol{e}_{3} \otimes \boldsymbol{e}_{1}\right), \\
\mathbb{F} \cdot \boldsymbol{e}_{2}=\frac{C}{h}\left(\boldsymbol{e}_{2} \otimes \boldsymbol{e}_{3}+\boldsymbol{e}_{3} \otimes \boldsymbol{e}_{2}\right), \\
\mathbb{F} \cdot \boldsymbol{e}_{3}=\frac{C}{h}\left(\boldsymbol{e}_{1} \otimes \boldsymbol{e}_{1}+\boldsymbol{e}_{2} \otimes \boldsymbol{e}_{2}+2 \boldsymbol{e}_{3} \otimes \boldsymbol{e}_{3}\right),
\end{array}\right.
$$

where constant $C$ is not null and depends on the choice of the kernel function $W$. For example, if $W$ is the Gaussian kernel $C=K /(4 \sqrt{\pi})$ in three dimensions and $C=K /(3 \sqrt{\pi})$ in two dimensions. The components of the tensor $\mathbb{F}$ given by the array of Eqs. (B3) can be rewritten in a more meaningful form as

$$
\left\{\begin{array}{l}
\mathbb{F} \cdot \boldsymbol{\tau}=\frac{C}{h}(\boldsymbol{\tau} \otimes \boldsymbol{n}+\boldsymbol{n} \otimes \boldsymbol{\tau}), \\
\mathbb{F} \cdot \boldsymbol{n}=\frac{C}{h}(\mathbb{1}+\boldsymbol{n} \otimes \boldsymbol{n}) .
\end{array}\right.
$$

As a consequence, the smoothed viscous term (B1) normal and tangential components are

$$
\begin{gathered}
\langle\nabla \cdot \mathbb{V}\rangle \cdot \boldsymbol{\tau}=\frac{\mu 2 C}{h} \boldsymbol{\tau} \cdot \mathbb{D} \cdot \mathbf{n}, \\
\langle\nabla \cdot \mathbb{V}\rangle \cdot \boldsymbol{n}=\frac{\mu C}{h}[\operatorname{tr}(\mathbb{D})+\boldsymbol{n} \cdot \mathbb{D} \cdot \boldsymbol{n}] .
\end{gathered}
$$

The tangential stress boundary condition (2.8) for an incompressible Newtonian fluid, namely $\boldsymbol{\tau} \cdot \mathbb{D} \cdot \boldsymbol{n}=0$, can be applied to equality (B5). It implies the vanishing of the tangential component of the smoothed viscous term:

$$
\langle\nabla \cdot \mathbb{V}\rangle \cdot \tau=0 .
$$

Regarding the normal component (B6), the incompressibility assumption (tr $\mathbb{D}=0$ ) implies that

$$
\langle\nabla \cdot \mathbb{V}\rangle \cdot \boldsymbol{n}=\frac{\mu C}{h} \boldsymbol{n} \cdot \mathbb{D} \cdot \boldsymbol{n} .
$$

Since $C \neq 0$ this normal component of the smoothed stress tensor vanishes if and only if $\boldsymbol{n} \cdot \mathbb{D} \cdot \boldsymbol{n}=0$. Consequently, when $\boldsymbol{n} \cdot \mathbb{D} \cdot \boldsymbol{n} \neq 0$ at the free surface, the normal component of the smoothed stress tensor $\langle\nabla \cdot \mathbb{V}\rangle$ diverges like $O(1 / h)$.

\section{APPENDIX C: DERIVATION OF THE DISSIPATION INTEGRAL EXPRESSIONS FOR THE MGF AND MEAF}

In the present appendix we study the dissipation integral expression:

$$
\int_{\Omega}\langle\nabla \cdot \nabla\rangle \cdot \boldsymbol{u} d V
$$

where $\langle\nabla \cdot \mathbb{V}\rangle$ is given by the MGF or the MEAF.

Let us assume the fluid has a free surface and no solid boundaries. Using the MGF Eq. (C1) becomes

$$
\mu K \int_{\Omega} d V \int_{\Omega} \frac{\left(\boldsymbol{u}^{\prime}-\boldsymbol{u}\right) \cdot\left(\boldsymbol{r}^{\prime}-\boldsymbol{r}\right)}{\left|\boldsymbol{r}^{\prime}-\boldsymbol{r}\right|^{3}} \boldsymbol{u} \cdot\left(\boldsymbol{r}^{\prime}-\boldsymbol{r}\right) \frac{\partial W}{\partial s} d V^{\prime}
$$

Writing $\boldsymbol{u}=\left(\boldsymbol{u}-\boldsymbol{u}^{\prime}\right) / 2+\left(\boldsymbol{u}+\boldsymbol{u}^{\prime}\right) / 2$ we decompose Eq. (C2) as follows:

$$
-\mu K \int_{\Omega} d V \int_{\Omega} \frac{\left[\left(\boldsymbol{u}^{\prime}-\boldsymbol{u}\right) \cdot\left(\boldsymbol{r}^{\prime}-\boldsymbol{r}\right)\right]^{2}}{\left|\boldsymbol{r}^{\prime}-\boldsymbol{r}\right|^{3}} \frac{\partial W}{\partial s} d V^{\prime}+\mu K \int_{\Omega} d V \int_{\Omega} \frac{\left(\boldsymbol{u}^{\prime}-\boldsymbol{u}\right) \cdot\left(\boldsymbol{r}^{\prime}-\boldsymbol{r}\right)}{\left|\boldsymbol{r}^{\prime}-\boldsymbol{r}\right|^{3}}\left(\boldsymbol{u}^{\prime}+\boldsymbol{u}\right) \cdot\left(\boldsymbol{r}^{\prime}-\boldsymbol{r}\right) \frac{\partial W}{\partial s} d V^{\prime}
$$

The kernel inside the second double integral is antisymmetric and, as a consequence of the double integration, gives a contribution which is identically null. Note that such a reasoning holds true only in absence of solid boundaries and/or interfaces with other fluids. Otherwise, the boundary influence should be added to the computations above invalidating the symmetry properties of the double integrals. With respect to the first double integral one can note that

$$
\left(\boldsymbol{u}^{\prime}-\boldsymbol{u}\right) \cdot\left(\boldsymbol{r}^{\prime}-\boldsymbol{r}\right)=\left[\left.\frac{\partial u_{i}}{\partial r_{j}}\right|_{r}\left(r_{j}^{\prime}-r_{j}\right)+\mathcal{O}\left(\left|\boldsymbol{r}^{\prime}-\boldsymbol{r}\right|^{2}\right)\right]\left(r_{i}^{\prime}-r_{i}\right)=\left(r_{i}^{\prime}-r_{i}\right) \mathbb{D}_{i j}\left(r_{j}^{\prime}-r_{j}\right)+\mathcal{O}\left(\left|\boldsymbol{r}^{\prime}-\boldsymbol{r}\right|^{3}\right),
$$

leading to

$$
\begin{aligned}
& \frac{\mu K}{2} \int_{\Omega} \mathbb{D}_{i j} \mathbb{D}_{k l} d V \int_{\Omega} \frac{\left(r_{i}^{\prime}-r_{i}\right)\left(r_{j}^{\prime}-r_{j}\right)\left(r_{k}^{\prime}-r_{k}\right)\left(r_{l}^{\prime}-r_{l}\right)}{\left|\boldsymbol{r}^{\prime}-\boldsymbol{r}\right|^{3}} \frac{\partial W}{\partial s} d V^{\prime}+\mathcal{O}(h) \\
& =\mu \int_{\Omega} \mathbb{D}_{i j} \mathbb{D}_{k l} \mathbb{S}_{i j k l} d V+\mathcal{O}(h)=-\mu \int_{\Omega}(\operatorname{tr} \mathbb{D})^{2} d V-2 \mu \int_{\Omega} \mathbb{D}: \mathbb{D} d V+\mathcal{O}(h)
\end{aligned}
$$

that is

$$
\int_{\Omega} \boldsymbol{u} \cdot\langle\nabla \cdot \mathbb{V}\rangle^{\mathrm{MG}} d V=-\mu \int_{\Omega}(\operatorname{tr} \mathbb{D})^{2} d V-2 \mu \int_{\Omega} \mathbb{D}: \mathbb{D} d V+\mathcal{O}(h)
$$

Using the MEAF and following the same procedure one obtains

$$
\int_{\Omega} \boldsymbol{u} \cdot\langle\nabla \cdot \mathbb{V}\rangle^{\mathrm{MEA}} d V=\mu \int_{\Omega} d V \int_{\Omega} \frac{\left|\boldsymbol{u}^{\prime}-\boldsymbol{u}\right|^{2}}{\left|\boldsymbol{r}^{\prime}-\boldsymbol{r}\right|} \frac{\partial W}{\partial s} d V^{\prime}=\mu \int_{\Omega}\left(\frac{\partial u_{i}}{\partial r_{j}}\right)\left(\frac{\partial u_{i}}{\partial r_{k}}\right) \mathbb{M}_{j k} d V+\mathcal{O}(h)=-\mu \int_{\Omega}\|\nabla \boldsymbol{u}\|^{2} d V+\mathcal{O}(h)
$$


[1] S. Mas-Gallic and P. Raviart, Numer. Math. 51, 323 (1987).

[2] R. Di Lisio, E. Grenier, and M. Pulvirenti, Ann. Scuola Norm. Sup. Pisa Cl. Sci. 24, 227 (1997).

[3] R. Di Lisio, E. Grenier, and M. Pulvirenti, Comput. Math. Appl. 35, 95 (1998).

[4] B. Ben Moussa and J. P. Vila, SIAM J. Numer. Anal. 37, 863 (2000).

[5] P. Español and M. Revenga, Phys. Rev. E 67, 026705 (2003).

[6] X. Hu and N. Adams, J. Comput. Phys. 213, 844 (2006).

[7] A. Colagrossi, M. Antuono, and D. Le Touzé, Phys. Rev. E 79, 056701 (2009).

[8] J. J. Monaghan and R. A. Gingold, J. Comput. Phys. 52, 374 (1983).

[9] J. P. Morris, P. J. Fox, and Y. Zhu, J. Comput. Phys. 136, 214 (1997).

[10] S. Marrone, A. Colagrossi, D. Le Touzé, and G. Graziani, J. Comput. Phys. 229, 3652 (2010).
[11] J. J. Monaghan, Rep. Prog. Phys. 68, 1703 (2005).

[12] J. Marsden and T. J. R. Hughes, Mathematical Foundations of Elasticity (Dover, New York, 1994).

[13] R. Glowinski and P. Le Tallec, Augmented Lagrangian and Operator-splitting Methods in Nonlinear Mechanics (SIAM, Philadelphia, Pennsylvania, 1989).

[14] J. Bonet and T. Lok, Comput. Methods Appl. Mech. Eng. 180, 97 (1999).

[15] A. Colagrossi, Ph.D. thesis, Università di Roma La Sapienza, Rome, Italy, 2005.

[16] J. Serrin, in Handbuch der Physik (Springer-Verlag, Berlin, 1959), Vol. VIII/1, pp. 125-263.

[17] J. Lighthill, Waves in Fluids (Cambridge University Press, Cambridge, England, 2001).

[18] M. Moakher, Q. J. Mech. Appl. Math. 61, 181 (2007).

[19] L. D. Landau and E. M. Lifshitz, Theory of Elasticity (Pergamon, London, 1959). 